\title{
Human Error Recovery for A Human/Robot Parts Conveyance System
}

\author{
YAMADA, Yoji, MORIZONO, Tetsuya, UMETANI, Yoji \\ Toyota Technological Institute, \\ 2-12-1, Hisakata, Tempaku, Nagoya 468-8511, Japan \\ yamada@toyota-ti.ac.jp \\ YAMAMOTO, Takahisa \\ Kojima Press Industry Co., Ltd., \\ 3-30 Shimoichiba-cho, Toyota, Aichi 471-8588, Japan
}

\begin{abstract}
The study concerns a human error recovery function for efficient human/robot collaborative parts conveyance tasks, which has not been discussed so far in construction of human/robot coexistence systems. We propose a human operational intention inference updater which allows a robot to judge whether the goal intended to reach by the operator is correct or not, from incomplete trajectory data sequence of the robot endtip maneuvered by the operator. Experimental results showed that a use of the proposed inference updater reduced the task execution time even when human error occurred in a task, which demonstrate the usefulness of the updater.
\end{abstract}

\section{Introduction}

We have developed human coexistence robots called "Skill-Assist" [1] which assist human workers in reflecting their skills, and introduced them to practical assembly lines. Figure 1 illustrates a simplified image of the Skill-Assist which has almost the same industrial mission as Cobots do [2], but an additional positioning mechanism and a control structure are installed.

In such human/robot coexistence systems, it is inevitable to maintain the system reliability for securing the safety of human operators and avoiding a lowering of task efficiency. Redundant mechanisms of enhancing the reliability of robots have been proposed in various aspects. A survey paper [3] introduces many redundant mechanisms on fault detection and tolerance schemes mainly focused on low-level robot controllers. Some other recent studies of interest include such proposals as: (a) a fault

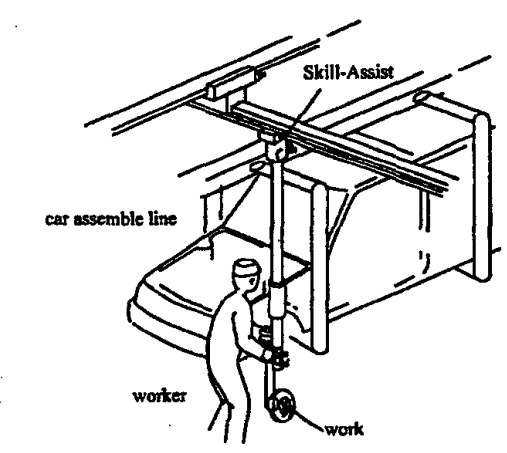

Figure 1: Illustration of Skill-Assist for parts assembly tasks

tolerant joint structure with redundant allocation of dual actuators for each joint of the space shuttle remote manipulator [4]. (b) failure-tolerant inverse kinematics schemes proposed for kinematically redundant robots experiencing locked joint failures [5]. (c)an approach to using commercial-off-theshelf products for designing a fault-tolerant bus architecture comprised of two different types of busses [6]. (d)construction of fully redundant (dual) signal process sequences under functional safety, starting from joint angle and endtip force sensors to the computation of dynamics and torque output for impedance control of a robot [7].

In the case of developing human/robot coexistence systems, however, we should not only pay our attention to the reliability of robot operation but also discuss the reliability on a human opera- 
tor's side [7]. In other words, there are two principal factors that may result in any faulty operation of the overall system; abnormal robot motions and errors committed by a human operator. Accordingly, it can also be said that there comes out a distinct possibility for both the operator and the robot sides to support each other as a redundant mechanism of maintaining the availability of the overall coexistence system. Figure 2 shows a general view of our experimental setup for the study, where the availability maintenance concept for the coexistence system comprising a human operator and a robot is illustrated by the outer frame arrows in view of a task life cycle.

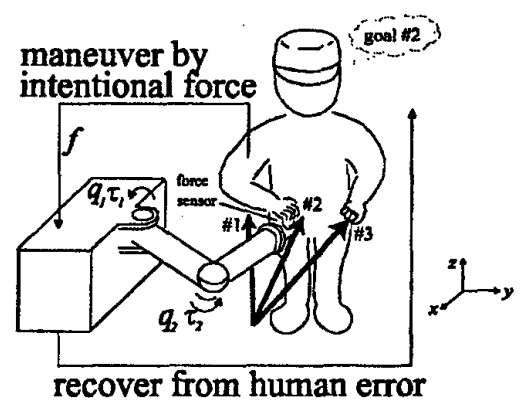

Figure 2: Experimental setup for the study and the availability maintenance concept

Regarding this direction, automated functions of detecting human erroneous action has also started to be reported: A force sensor signal was used for human error detection through development of a modified HMM algorithm [7], and the necessity of a human/robot agreement process was stressed in the study. Moreover, a proposal of hybrid HMMs and its application to detection of human sit-back and step failures in the operation of reconfigurable stand-up, seating, and mobile wheelchair [8]. Several studies refer to that human intention can also be inferred stochastically through human behavioral state transition [7][8][9]. However, there has been no proposal concerning recovery capability of the overall system from a viewpoint of availability recovery toward construction of dependable [10] human/robot coexistence systems.

As was mentioned earlier, even a human operator may commit an error. Therefore, it is desired that a robot which is the other component of the coexistence system plays a role of recovering the erroneous performance of the system initiated on the human operator's side.

In the paper, technical attention is paid to real- izing an error recovery function of the robot against human erroneous action to maintain the system productivity. We conceptually stress that the robot should watch any change in the human intention for operation $^{1}$ (abbreviated as ITO from InTention for Operation) toward the goal until the task is completed. We verify the capability of updating the human ITO inference for erroneous task recovery.

\section{Human ITO inference updater us- ing HMM}

\subsection{Overview of HMM modified for} temporal Human ITO inference

To handle trajectory data obtained from the operator's maneuvering movement, we employ Hidden Markov Model (HMM) to solve the problem of human motion rate variation. HMM basically solves three problems [11], but we need to solve two more problems for our study in addition to those three:

- Problem 4: In the original model, each pattern accepts only one trained sequence of data.

- Problem 5: Pattern matching function is conducted after all data of the observed sequence are presented.

In HMM, pattern \# $h$ is modeled as $\lambda_{h}$ by three probability measures as in (1); probability distribution of the state transition $\mathbf{A}=\left\{a_{i j}\right\}$ from $i$ to $j(1<i, j<N)$, the observation symbol probability distribution $\mathbf{B}=\left\{b_{j}(l)\right\}$ generating output $v_{l}$ in $j$-th state, and the initial state distribution $\pi_{h}=\left\{\pi_{h_{i}}\right\}$.

$$
\lambda_{h}=\left\{\mathbf{A}_{h}, \mathbf{B}_{h}, \boldsymbol{\pi}_{h}\right\} \quad h=1, \cdots, H
$$

For solving the above Problem 4, we need to take $k(1<k<K)$ times of observation sequences, each ending at time $T_{k}$, to reestimate the model parameters with the reestimation algorithm formulated as in (2) and (3).

$$
\begin{gathered}
\bar{a}_{i j}=\frac{\sum_{k=1}^{K} \sum_{t=1}^{T_{k}-1} \alpha_{t}^{k}(i) a_{i j} b_{j}\left(o_{t+1}^{k}\right) \beta_{t+1}^{k}(j)}{\sum_{k=1}^{K} \sum_{t=1}^{T_{k}-1} \alpha_{t}^{k}(i) \beta_{t+1}^{k}(i)} \\
\sum_{j=1}^{K} \sum_{t=1}^{T_{k}-1} \alpha_{t}^{k}(j) \beta_{t+1}^{k}(j) \\
\sum_{k=1}^{K} \sum_{t=1}^{T_{k}-1} \alpha_{t}^{k}(j) \beta_{t+1}^{k}(j)
\end{gathered}
$$

\footnotetext{
${ }^{1}$ In the study, the meaning of intention is limited to an operator's intention of completing geometrically-fixed conveyance tasks.
} 
from which forward and backward algorithms are conducted using $\alpha_{t}^{k}(i)$ and $\beta_{t}^{k}(i)$ respectively which are defined as follows.

$$
\begin{aligned}
\alpha_{t}^{k}(i) & =P\left(o_{1}{ }^{k} o_{2}{ }^{k} \cdots o_{t}{ }^{k}, s_{t}=S_{i} \mid \lambda\right) \\
\beta_{t}^{k}(i) & =P\left(o_{t+1}^{k} o_{t+2}^{k} \cdots o_{T}^{k} \mid s_{t}=S_{i}, \lambda\right)
\end{aligned}
$$

The solution to the Problem 5 which associates us with a temporal inference function is given by (4), because we need the probability that the output $\mathbf{O}_{t}=o_{1} \cdots o_{t}$ is generated and while the state is staying at $s_{t}=S_{i}$ (only constrained at $t$ ) under model $\lambda_{h}$.

$$
P\left(\mathrm{O}_{t} \mid \lambda_{h}\right)=\sum_{j=1}^{N} \alpha_{t_{h}}(j)
$$

which is proved iteratively by:

$$
\sum_{j=1}^{N} \alpha_{t_{h}}(j)=\sum_{j=1}^{N}\left[\left\{\sum_{i=1}^{N} \alpha_{t-1_{h}}(i) a_{i j}\right\} b_{j}\left(o_{t}\right)\right]
$$

After the above modified HMM algorithm is formulated, the human ITO inference probability of being matched as pattern $\# h$ is computed at each sampling period by the following percentage:operation.

$$
p_{h}(i) \equiv \frac{P\left(\mathbf{O}_{t} \mid \lambda_{h}\right)}{\sum_{h=1}^{H} P\left(\mathbf{O}_{t} \mid \lambda_{h}\right)}
$$

\subsection{Human ITO Inference Updating Algorithm}

The HMM algorithm described in the last subsection allows the robot to infer to which set position an operator tries to maneuver the robot arm even before the robot endtip reaches the set position. This implies that the algorithm is applicable to human error detection in the early stage of inferring the human-intended path pattern. Accordingly, the robot is possibly given a function to alarm the operator for correcting his/her error so that he/she notices it later but still before the task is completed. The possibility offers a chance to the operator for appropriately changing the conveyance trajectory toward a new goal. To achieve the above correction capability, we need to extend the function of temporally inferring human ITO even if it is changed in the course of the task. We refer to the extended inference function as an inference updater.

The basic idea of achieving this inference updating function is as follows: When a change in the human ITO is detected at point $\mathbf{P}_{\boldsymbol{i}}$, the next candidates $\mathbf{P}_{\boldsymbol{o}}$ which are computed as being the closest from $\mathbf{P}_{\boldsymbol{i}}$ in the robot endtip motion direction can be inferred as new operationally-intended trajectories. According to the computation of the operational goal inference, we can hypothetically draw a line $\overrightarrow{\mathbf{P}_{i} \mathbf{P}_{o}}$ as a virtually trained path (abbreviated as VTP) data in the form of a discrete HMM. Naturally, we need to accept inherently curved trajectories of human arm voluntary movements [12], and in the study, the trajectory in one trial differs from those in the others because the moment when the worker changes his/her ITO cannot be fixed. For this problem, we propose to draw VTPs whose trajectories vary each other with a Gaussian distribution. Practically, the re-estimation procedure in HMM, which was introduced in section 2 computes $\bar{a}_{i j}$ and $\bar{b}_{j}(l)$ using the data $\mathrm{O}_{t}{ }^{k}$ which are composed of VTPs with erroneous distance distribution $\Delta d$ deviated from the VTP with 0 mean and $\sigma^{2}$ variance.

$$
P(\Delta d)=\frac{1}{\sqrt{2 \pi} \sigma} e^{-\frac{(\Delta d)^{2}}{2 \sigma^{2}}}
$$

Then, the HMM reestimation algorithm formulated in (2) and (3) is driven for the multiple VTP data sequences.

\subsection{Verification of the ITO inference update}

We have already demonstrated the effectiveness of the basic technique for the temporal humanintended path pattern inference [13]. In this section, we verify that the inference updater of our proposal functions adequately by showing a result of inferring human ITO changes in the course of a task. We drew a resultant robot endtip motion trajectory with 2-time human ITO changes supposed as shown (on the left-hand side) in Figures 3(aL), (bL), and (cL). The curved line -.. shows data sequence along an actually sampled motion trajectory. In the beginning of the maneuvering motion, 3 VTPs (with Gaussian distribution) are drawn and the velocity vector of the trajectory indicates that the human ITO is toward set position \#3. It is verified by the inference result from $x=0.2$ to $x=0.1$ in Figure 3(aR). In the figure (bL), there occurs a human ITO change at $x=0.1 \mathrm{~m}$. From this point, new VTPs are drawn toward set positions \#1 through \#3 as shown in the same figure. It is seen from Figure (bR) that a new human ITO directing to set position \#1 has been inferred. Similarly, the next change of human ITO happens again 
at $x=-0.1 m$ in Figure 3(cL), and the result of updating human ITO inference in Figure 3(cR) shows the correct answer.

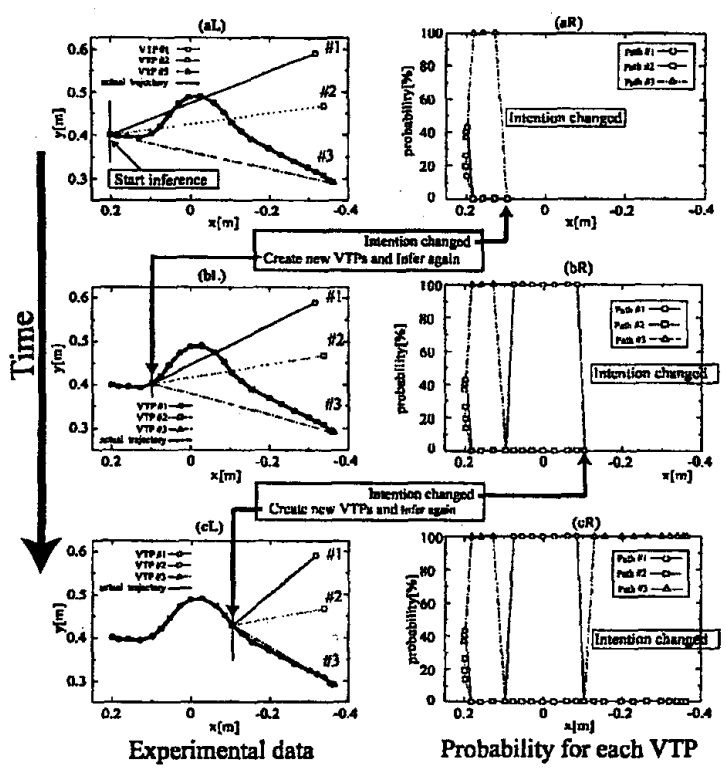

Figure 3: Experimental results of the robot endtip trajectory drawn by the human maneuvering motion with VTPs (left side) and the human ITO inference updating results corresponding with geometrical trajectory course changes (right).

\section{Verification of human error recov- ery function in parts conveyance tasks}

In this section, we present the next experiments for demonstrating effectiveness of the human error recovery function which is the main issue of this paper. The recovery function is driven by applying the human ITO inference updater described in 2.2 .

\subsection{Task Experiments}

Parts conveyance and assortment tasks in the experimental human/robot coexistence system are adopted for the demonstration. The task contents are to pick up and to convey one of the numerically labeled 27 parts with a power assistance of the robot, and to position the part according to the number to either one of the three set positions. One task is counted to be finished if a part is successfully placed at one of the correct set positions which are circles with a radius of $50 \mathrm{~mm}$, and the predetermined number is matched with the label number of the part. 9 parts multiplied by 3 times, i.e. 27 parts in all are assigned to a subject to complete a job. This is a simplified example of parts assort- ment tasks in practical parts assembling lines with robots introduced to coexist with human workers. Note that each of the tasks has redundant solutions to where a part is to be assorted as shown in Figure 4 where there are 4 kinds of labels on the 27 parts. The operator can plan an efficient process solution making use of the redundancy.

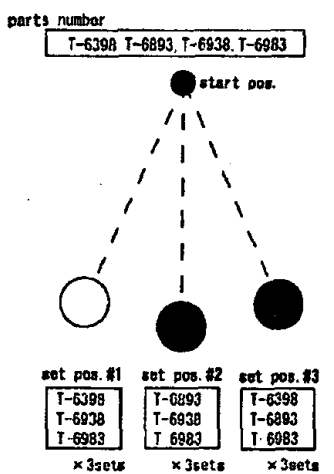

Figure 4: Conveyance task conditions with a 4-digit label on each part

At the task planning stage,

(1) Each subject is given 5 minutes to learn the job rule, i.e. the relation between the label numbers on parts and the numbers assigned at the set positions. ${ }^{2}$

(2) The subject is allowed to map out a strategy for efficiently completing the job composed of 27 tasks.

At the next practical job execution stage,

(3) The subject is asked to pick up a part located at the start position which is set at $x=0.2$ and $y=0.4$ in the coordinate frame defined in Figure 2 and to look at the 4-digit label number on the display. Then he/she brings the part by maneuvering the handle at the robot endtip to one of the set positions for placing the part.

(4) The subject is given at most 3 seconds to complete each task, and he needs to complete 27 correct tasks.

At the job execution stage, the robot is programmed to light all of the 3 colors of an alarm signal mounted on the robot trunk, and to admonish the subject of the necessity for a change of his/her ITO. When a part is finally reached a correct set

\footnotetext{
${ }^{2}$ In practical production lines, 4-digit numbers are frequently used for parts classification, which sometimes leads misunderstandings to cause human lapse.
} 
position, the robot displays only a blue color of the signal to show that the task is successfully completed. Figure 5 illustrates typical two supposed cases of trajectories that would be likely to appear in a task. Figure 5(a) shows a path taken by a subject without the proposed inference updater: a subject brings a part to a set position and changes the direction of the robot endtip to another set position after being informed by the robot that the original position is incorrect, and so on. On the other hand, figure 5(b) shows a more efficient path course with an alarming assistance of the inference updater that the initially taken path up to a halfway position is going to be incorrect. The subject tries changing the path course toward the next position of proposal in correspondence with the alarm from the robot.
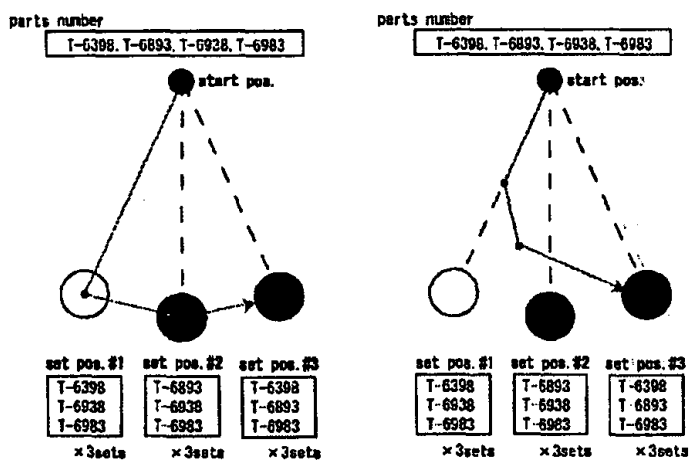

Figure 5: Two comparative cases of motion trajectories (a) without and (b) with the human ITO inference updater.

\subsection{Experimental results}

Table 1 shows some results of the experiments conducted by 3 men out of 7 who are engaged in the engineering-based research. The probability that each of the subjects initially takes erroneous paths scores $10 \%$ for subject $A, 16 \%$ for $B$, and $18 \%$ for C. When we look at the 13th task of the subject $A$, he is about to bring the 13 th part to \#2 set position in the beginning though the correct position is either \#1 or \#3. It is also shown in the same row that after he was alarmed to change the path course, he took the next proposal from \#2 to \#3. It took only $1.3 s$ to complete the task with the inference updater employed ( $\mathrm{Ha}$ ), while $2.6 \mathrm{~s}$ was taken without the updater (\#b) which was conducted on purpose afterward. Figure 6 traces the change of the human ITO in the above case of the 13th part. The results listed in the table demonstrate the usefulness of the proposed human ITO inference updater in terms of total task execution time. Therefore, we could demonstrate that the updater effectively maintained task efficiency of the system against the human erroneous actions.

Interviews were made to the subjects when they committed errors after their task completion. Earlier, it was anticipated that numerical misperception of the 4-digit labels would be the only cause to draw human enormous action. Interestingly, however, the results of the interviews revealed that skill-based slips and lapses were also identified to be the causes in practice.

Table 1: Sample results of demonstrating the usefulness of the human ITO inference updater
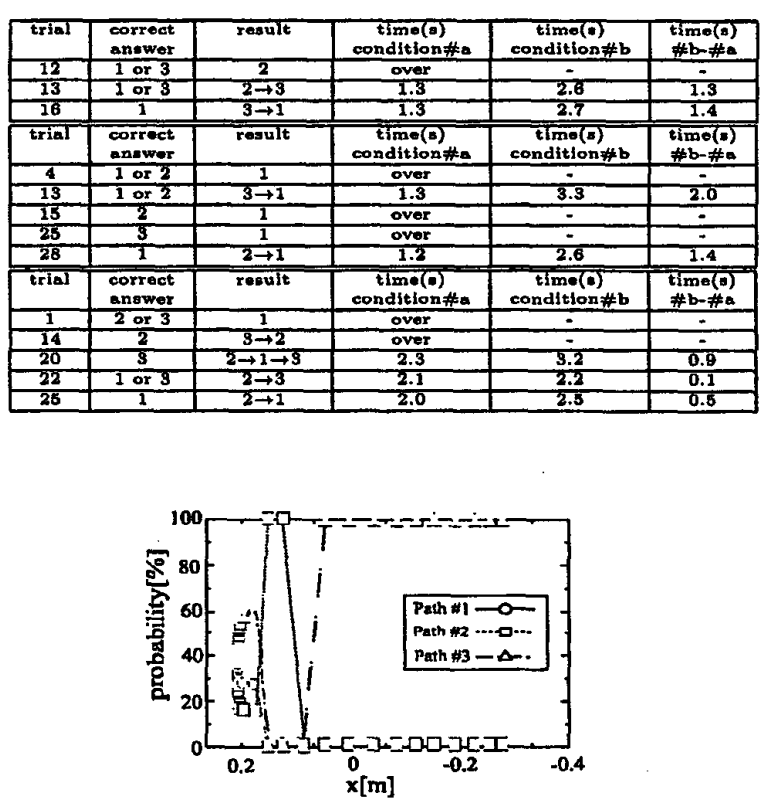

Figure 6: Result of updated human ITO inference in the case of the 13th part for subject $A$.

\section{Conclusions}

In pursuit of a highly maintenable coexistence system, the paper described a useful human error recovery function for efficient human/robot collaborative parts conveyance tasks. We proposed to update the result of inferring human operational intention. The paper is summarized in the following 3 items.

(1) We discussed availability maintenance of a coexistence system from a viewpoint of human/robot dependability: The robot side recovers erroneous performance of the system 
caused by the human operator, using the robot's capability of inferring the operational intention.

(2) We defined the operational intention inference capability which was characterized by the ability of inferring the goal of the human motion in the early stage from a geometrical data sequence of a fixed task motion. We modified the HMM algorithms for our target. Moreover, generation of virtual trained paths which connect the current position and the next candidate set positions allows us the function of updating the human operational intention inference.

(3) To demonstrate the usefulness of the inference updater, we conducted some experiments in which subjects conveyed parts with 4-digit label numbers to either one of the appropriate set positions based on the predetermined task conditions while maneuvering the robot endtip. Experimental results were listed to show that a use of the proposed inference updater reduced the task execution time, which proved that the updater was available and maintained task efficiency against human erroneous action.

The experimented task condition was set to be fairly easy compared with the practical ones. Though we still need to analyze and model practical task sequences more intensively, we consider that the concept proposed in the paper has wide applicability in practice from viewpoints of not only productivity but also safety, if the dimension and resolution of state space segmentation are properly reconfigured.

\section{Acknowledgment}

The authors are indebted to Mr. SAKAI, Takayuki at Toyota Motor Corporation for his work on HMM implementation. This study was partly supported by the Ministry of Education, Culture, Sports, Science and Technology under Grant-in-aid for Scientific Research No. 09221225.

\section{References}

[1] Yoji YAMADA et al., Proposal of Skill-Assist: A system of Assisting Human Workers by Reflecting Their Skills in Positioning Tasks, Proc. of IEEE Int. Conf. on Systems, Man, and Cybernetics (SMC '99), Tokyo, pp.IV11-16, 1999
[2] P. Akella et al., Cobots for the automobile assembly line, Proc. of 1999 Int. Conf. on Robotics and Automation, Detroit, pp.728-733, 1999

[3] M. L. Vinsky et al., Robotic fault detection and fault tolerance: A survey, Reliability Engineering and Systems Safety, Vol.46, pp.139-158, 1994

[4] Eugene C. Wu et al., Fault-Tolerant Joint Development for the Space Shuttle Remote Manipulator System: Analysis and Experiment, IEEE Trans. on Robotics and Automation, Vol.9, No.5, pp.675684, October 1993

[5] M. Goel et al., The Design of Control Strategies Tolerant to Unidentified Failures in Kinematically Redundant Manipulators, Proc. of 1999 Int. Conf. on Robotics and Automation, Detroit, pp.867-873, 1999

[6] S. N. Chau et al., Design of a Fault-Tolerant COTS-Based Bus Architecture, IEEE Trans. on Reliability, Vol. 48, No. 4, pp. 351-359, 1999

[7] YAMADA Yoji et al., FTA-Based Issues on Securing Human Safety in a Human/Robot Coexistence System, Proc. of IEEE Int. Conf. on Systems, Man, and Cybernetics (SMC '99), Tokyo, pp.II1058-1063, 1999

[8] M. Hiratsuka et al., Detection of Human Mistakes and Misperception for Human Perceptive Augmentation: Behavior Monitoring Using Hybrid Hidden Markov Models, Proc. of 2000 IEEE Int. Conf. on Robotics and Automation, San Francisco, pp. 577$582,2000$.

[9] Taketoshi MORI et al., Situation Reactive Handiwork Support through Behavior Understanding, Proc. of the 1997 IEEE Int. Conf. on robotics and Automation, New Mexico, pp.587-592, 1997.4

[10] Algirdas Avizienis et al., Fundamental Concepts of Computer System Dependability, Proc. of IARP/IEEE-RAS Workshop on robot Dependability: Technological Challenge of Dependable Robots in Human environments - Seoul, pp.I-1, 2001.5

[11] L. R. Rabiner, A Tutorial on Hidden Markov Models and Selected Applications in Speech Recognition, Proc. of IEEE, Vol.77, No.2, pp.257-286, 1989

[12] T. Flash et al., The Coordination of Arm Movements: An Experimentally Confirmed Mathematical Model, The Journal of Neuroscience, vol.5, No.7, pp.1688-1703, 1985

[13] YAMADA Yoji et al., Construction of a Human/Robot Coexistence System Based on A Model of Human Will - Intention and Desire, Proc. of the 1999 IEEE Int. Conf. on Robotics and Automation, pp.2861- 2867, 1991 